\title{
Structural Topology Optimization: Moving Beyond Linear Elastic Design Objectives
}

James K. Guest

Johns Hopkins University, jkguest@jhu.edu

Reza Lotfi

Johns Hopkins University

Andrew T. Gaynor

Johns Hopkins University

Mehdi Jalalpour

Cleveland State University, m.jalapour@csuohio.edu

Follow this and additional works at: https://engagedscholarship.csuohio.edu/encee_facpub

Part of the Civil Engineering Commons, and the Structural Engineering Commons

How does access to this work benefit you? Let us know!

\section{Original Citation}

Guest, J.K., Lotfi, R., Gaynor, A., Jalalpour, M., "Structural topology optimization - moving beyond linear elastic design objectives", Structures Congress 2012; March 29-31, 2012, Chicago, Illinois, p. 245-256.

This Conference Proceeding is brought to you for free and open access by the Civil and Environmental Engineering at EngagedScholarship@CSU. It has been accepted for inclusion in Civil and Environmental Engineering Faculty Publications by an authorized administrator of EngagedScholarship@CSU. For more information, please contact library.es@csuohio.edu. 


\title{
Structural Topology Optimization: Moving Beyond Linear Elastic Design Objectives
}

\author{
James K. Guest, Reza Lotfi, Andrew T. Gaynor, Mehdi Jalalpour
}

Department of Civil Engineering, The Johns Hopkins University, 3400 North Charles

Street, Baltimore, MD 21218; PH (410) 516-3923; FAX (410) 516-7473; jkguest@jhu.edu

\begin{abstract}
Topology optimization is a systematic, free-form approach to the design of structures. It simultaneously optimizes material quantities and system connectivity, enabling the discovery of new, high-performance structural concepts. While powerful, this design freedom has a tendency to produce solutions that are unrealizable or impractical from a structural engineering perspective. Examples include overly complex topologies that are expensive to construct and ultra-slender subsystems that may be overly susceptible to imperfections. This paper summarizes recent tools developed by the authors capable of mitigating these shortcomings through consideration of (1) constructability, (2) nonlinear mechanics, and (3) uncertainties.
\end{abstract}

\section{INTRODUCTION}

The potential for topology optimization as a powerful, free-form design tool has long been demonstrated in literature. The freedom to add (or remove) material resources to (or from) any point in the design domain means material quantities and system connectivity are simultaneously optimized - a powerful concept. While this design freedom enables the discovery of high performance solutions, it often leads to structural designs that are impractical from engineering and/or construction points of view, or that are overly sensitive to uncertainties such as imperfections or random loads. These flaws tend to be amplified by the fact that most topology optimization works consider design objectives and specifications associated with linear elastic structural behavior. This paper presents recent efforts by the authors for mitigating these shortcomings. Specifically, methodological and algorithmic tool development to optimize structural topology for nonlinear structural behavior, constructability objectives, and for robustness in the presence of uncertainties.

The first step in topology optimization is discretization of the design domain. This meshing must ultimately be driven by the governing mechanics, but generally uses either solid (continuum) finite elements or discrete members such as truss or frame elements. In continuum topology optimization, the goal is to determine the optimal phase composition in each elemental domain. In traditional solid-void design, each element carries an indicator variable $\rho^{e}$, known as the volume fraction, that identifies the element as either solid material $\left(\rho^{e}=1\right)$ or a void $\left(\rho^{e}=0\right)$. The connectivity of the solid elements defines the topology of the structure. Such an 
approach may be appropriate, for example, in the design of concrete or steel components. Discrete element topology optimization, on the other hand, typically follows a ground structure approach, where the domain is discretized with a dense, redundant structural system and members deemed inefficient are removed from this ground structure.

While straightforward in concept, topology optimization problems are fundamentally and numerically challenging to solve. This has led the vast majority of research to focus on linear elastic and deterministic mechanics. The most commonly solved topology optimization formulation is minimum compliance, where the goal is to minimize internal strain energy (maximize stiffness) in a structure of fixed mass for given load case and boundary conditions. A design example of a simply supported beam loaded at midspan is shown in Figure 1. A simple design containing six uniformly spaced holes is shown in Figure $1 \mathrm{~b}$. This design features a 50\% volume fraction and undergoes a vertical deflection $d$ at midspan. Figure 1c displays a topology-optimized solution using the same volume of material but offering an increased stiffness (reduced deflection) of approximately $42 \%$. The design follows the principal stress trajectories with compression and tension members lying orthogonal. Alternatively, Figure 1d displays a solution having the same stiffness as the simple beam with regular holes (deflection $d$ ) but at a reduced weight of approximately $50 \%$.

While these improved performance metrics are compelling, the optimized topologies clearly illustrate the deficiencies that have inhibited the application of this tool to structural engineering. The topology in Figure 1c is certainly more challenging to fabricate than the solution in Figure 1b, while the topology in Figure 1d may offer the same elastic stiffness as the simple design but will more readily enter the nonlinear regime due to slender compressive load paths and will be more susceptible to flaws.

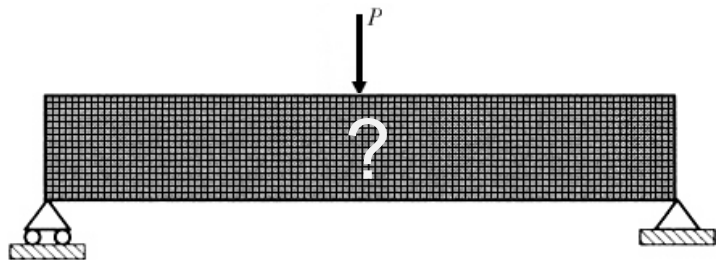

(a) Design Domain

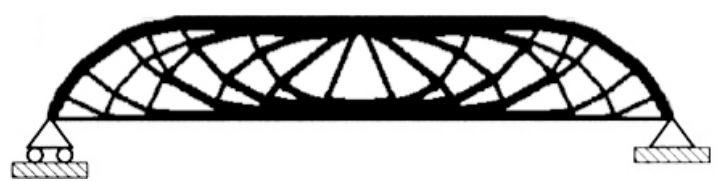

(c) Optimized Topology:

Weight $=W$, max deflection $\sim 0.58 d$

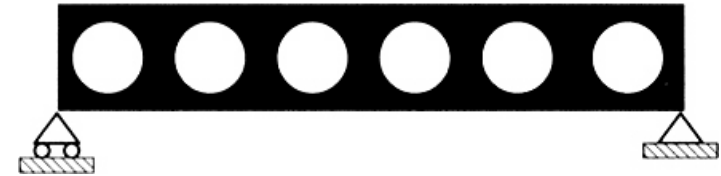

(b) Simple Design: Weight $=W$, max deflection $=d$

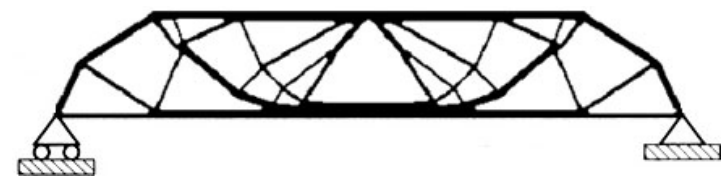

(d) Optimized Topology: Weight $\sim 0.50 \mathrm{~W}$, max deflection $=d$

Figure 1. Simply supported beam example. (a) Design domain and continuum mesh, (b) simple 50\% volume fraction design with uniformly spaced holes, (c) topology optimized design offering same weight as (b) but offering $42 \%$ improved stiffness, and (d) topology optimized design offering same linear elastic stiffness as (b) but at half the weight. 


\section{OPTIMIZATION FORMULATION: LINEAR ELASTIC MECHANICS}

We begin with a brief review of the linear elastic minimum compliance formulation, where the goal is to maximize stiffness of a fixed-mass system by minimizing internal strain energy or equivalently external work. The independent design variable for the topology optimization problem is denoted as $\rho^{e}$ and represents element cross-sectional areas in truss topology optimization and element volume fractions, in continuum domains. The response nodal displacements $\mathbf{d}$ are the state (dependent) design variables, and are related to the design through the equilibrium conditions. The design problem is given in general as

$$
\begin{array}{ll}
\min _{\boldsymbol{\rho}^{\mathrm{e}}, \mathbf{d}} & \frac{1}{2} \mathbf{d}^{\mathrm{T}} \mathbf{K}\left(\boldsymbol{\rho}^{\mathbf{e}}\right) \mathbf{d}=\frac{1}{2} \mathbf{f}^{\mathrm{T}} \mathbf{d} \\
\text { subject to : } & \mathbf{K}\left(\boldsymbol{\rho}^{\mathbf{e}}\right) \mathbf{d}=\mathbf{f} \\
& \sum_{e \in \Omega} \rho^{e} v^{e} \leq V \\
& 0 \leq \rho^{e} \leq \rho_{\max }^{e} \quad \forall e \in \Omega
\end{array}
$$

where $\mathbf{f}$ are the nodal applied loads, $v^{e}$ is element volume for unit $\rho^{e}$ (element length for truss structures), $V$ is the available volume of material, and $\rho_{\max }^{e}$ is the upper bound on $\rho^{e}$. The global stiffness matrix $\mathbf{K}$ is assembled in the usual manner from element stiffness matrices $\mathbf{K}^{\mathbf{e}}$, which are related to the design variables as follows:

$$
\mathbf{K}^{e}\left(\rho^{e}\right)=\left(\rho^{e}\right)^{\eta} \mathbf{K}_{0}^{e}
$$

where $\mathbf{K}_{0}^{\mathrm{e}}$ is the element stiffness matrix for unit $\rho^{e}$ and the exponent parameter $\eta \geq 1$ is an optional penalty term that may be used to drive solutions to the design variable bounds (Bendsøe 1989). The sensitivity analysis is performed using the adjoint method (see e.g., Bendsoe and Sigmund (2003)) and Method of Moving Asymptotes (MMA) (Svanberg 1987) is used in this work as the gradient-based optimizer.

\section{CONSTRUCTABILITY}

Maintaining constructability of topology-optimized solutions is critical to ensuring designs are cost effective and useable. The primary challenge here is quantifying constructability and, in the case of continuum design, computationally detecting structural members, or features, which are defined by the union of elements of like phase. Significant progress has been made in this arena through geometric restriction of the design space. For example, by controlling the minimum length scale (diameter) of designed members (Guest et al. 2004, Guest 2009a), maximum length scale of members (Guest 2009b), and pattern repetition (Stromberg et al. 2011). By tightening these restrictions, the designer is able to reduce design complexity and enhance constructability. 
This idea has been demonstrated in building design concepts (Stromberg et al. 2010) and, at the $19^{\text {th }}$ Analysis and Computation Specialty Conference, the optimization of concrete reinforcement patterns via strut-and-tie modeling (Guest and Moen 2010). We focus here on the latter, where topology optimization can be used to idealize the flow of forces in concrete members and automate generation of strut and tie models, where resulting tensile zones indicate regions for steel reinforcement or prestressing placement (Schlaich, 1987). For example, we consider the concrete hammerhead pier design problem presented in Guest and Moen (2010) where it was demonstrated that allowing smaller diameters of structural members leads to stiffer structures, but structures with more complex reinforcing steel geometries. This is shown in Figure 2, where the number of steel ties decreases from nine to five when the minimum allowable diameter increases from $r$ to $6 r$. This trend increases the steel material cost but reduces the number of reinforcing members to be placed, thereby lowering labor cost. Although difficult to quantify, the trends are evident.

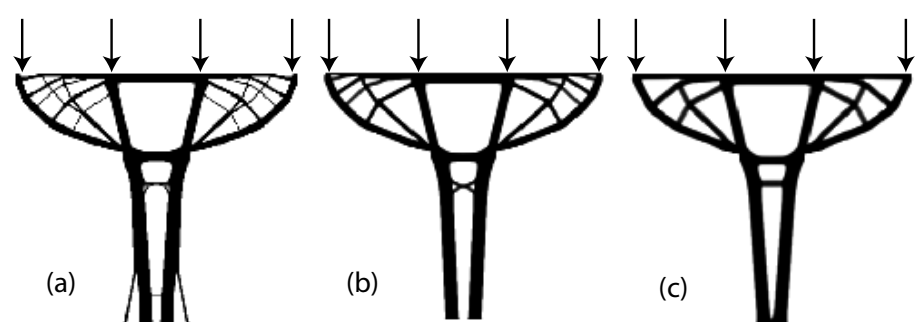

Figure 2. Hammerhead pier example solved continuum topology optimization and minimum prescribed length scales of (a) $r$, (b) $4 r$, and (c) $6 r$. Smaller length scales offer higher performance but typically increase design complexity

(Guest and Moen 2010).

A limitation of this idea, however, is that the shape of the reinforcement remains generally unconstrained (e.g., Liang et al. 2000, Bruggi 2009). In other words, reinforcement bars may be curved as seen in Figure 2. Such a design may be acceptable in the case of prestressing strands, but may be problematic or expensive for use with steel rebar. To circumvent this issue, we propose here a hybrid approach, which combines the truss and continuum topology optimization approaches. The idea is that steel reinforcement, which needs to have simple layouts and straight members in order to be cost efficient, is better idealized using truss elements. The concrete in the strut and tie model is an idealization of the flow of compressive forces, and thus need not be explicitly 'constructed', allowing geometric restrictions to be relaxed. Continuum elements are therefore used to model the concrete phase.

A bilinear constitutive model is adopted where truss elements offer high tensile stiffness but low compressive stiffness, while continuum elements offer high compressive stiffness but low tensile stiffness, a valid assumption if the concrete in tension is assumed cracked. The concrete is idealized using the model suggested by Darwin and Pecknold (1977). To achieve this effect, the design domain is discretized with a lattice mesh of nodes. The continuum mesh uses every node, while the truss mesh is more sparse with members connected at every few nodes in order to reduce complexity of the final steel configuration. This is seen below in Figure 3 where there 
are 12 continuum elements in each direction, but the truss elements are connected every 4 nodes (green continuum elements, blue truss elements). Force transfer between the meshes occurs at the shared nodes, and a nonslip condition for the steel reinforcement is assumed.

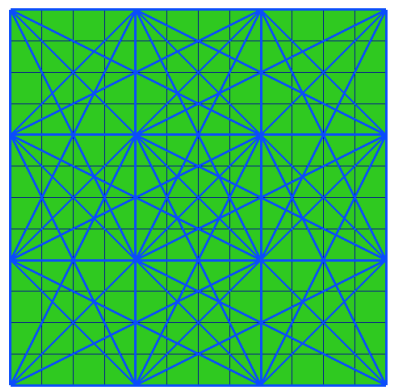

Figure 3. Interaction between continuum and truss domains

The following are some preliminary results from the hybrid optimization. The first example is a simply supported beam with a point load at the center, where symmetry has been used to reduce the computational cost (Figure 4). As can be seen, the tension steel is located in the bottom portion of the beam and is located along the principal stress trajectories. This is also seen in Figure 1, with the noticeable difference here being that the tensile members are sparse and straight, as would be desired to reduce labor costs.
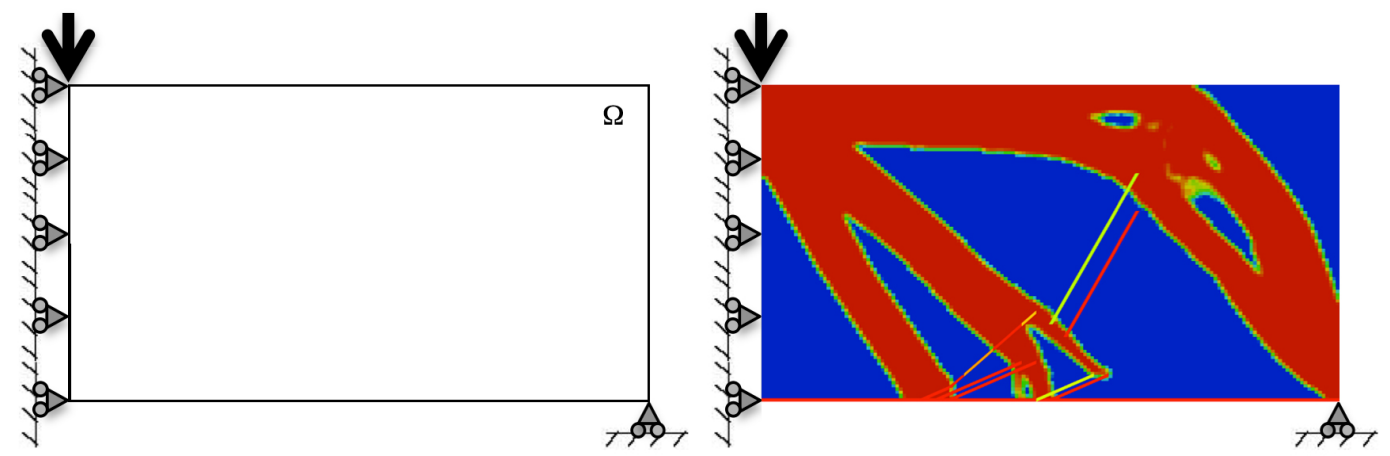

Figure 4. Simply supported beam design domain (left) and optimized strut and tie topology (right)

Topology optimization is especially useful for more complex geometries, where the flow of forces is difficult to visualize, let alone optimize. Figure 5 shows a modified dapped end beam with a hole in the middle that presents such a complication. The optimal solution is shown in Figure 5, where again the concrete compression zones are represented by the continuum structure and the tensile reinforcement pattern is restricted to be sparse and composed of straight members. While these examples highlight the capability of topology optimization to create strut-and-tie models from scratch, this tool can also be used to 'reinforce' a traditional, uniform rebar pattern. One would simply include the desired reinforcement pattern as a subset of the truss mesh and keep the associated crosssectional areas fixed (or bounded) to maintain their existence throughout the optimization. 

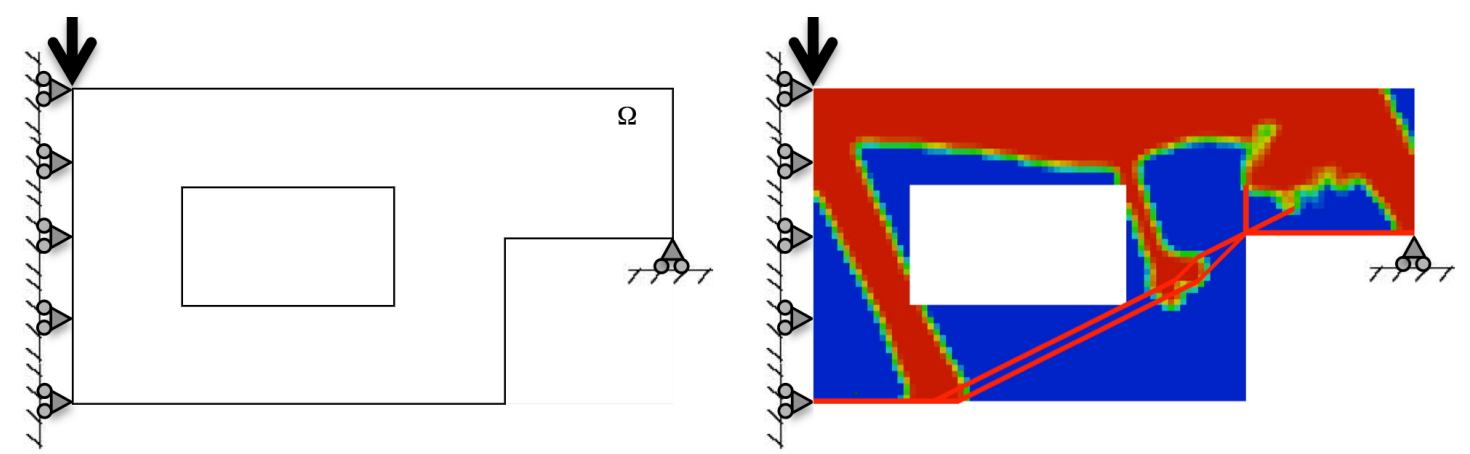

Figure 5. Modified dapped end beam with hole design domain (left) and optimized strut and tie topology.

\section{NONLINEAR BEHAVIOR}

Extending topology optimization to design under nonlinear mechanics is another challenging but important endeavor, particularly given the increasing use of performance-based design methodologies. As a first step, several researchers have proposed using linearized eigenstability metrics for optimizing structural stiffness (see e.g., Rahmatalla and Swan, 2003). Formal incorporation of geometric and/or material nonlinearities requires iterative analysis and a constraint on the residual $\mathrm{R}$ of equilibrium equations. Final compliance, cumulative compliance, and complimentary elastic work are have been suggested as objective functions by Buhl et al (2000). The drawback of final compliance as the objective function is that the structure may collapse under the load before reaches the end point. Cumulative compliance as the objective function is defined as

$$
\min f=\iint_{\Omega} \sigma^{T} d \varepsilon d \Omega
$$

where it should be evident that the nonlinear optimization is now a path dependent problem.

We begin by considering geometric nonlinearities. The residual is defined as

$$
R(U)=P-\int B^{T} \sigma d V
$$

Strains are related to the displacements as follows

$$
d \xi=B(U) d(U)
$$

where we use advanced B matrix to get higher order strains for Green-Lagrangian strains

$$
\xi_{i j}=\frac{1}{2}\left(u_{i, j}+u_{j, i}+u_{k, i} u_{k, j}\right)
$$


This system of nonlinear equations is incorporated into the finite element and sensitivity analyses and is solved using displacement control iterative method. To demonstrate the effect of geometric nonlinearity on optimal designs, consider the simple cantilever structure shown in Figure 6. We optimize for cumulative compliance under various load magnitudes. It is clearly seen that considering geometric nonlinearities leads to solutions that are load-dependent and, potentially, asymmetric. The latter can be understood by examining the structural member at the tip of the beam in Figures $7 \mathrm{~b}$ and $7 \mathrm{c}$. As the beam deflects, this member will become vertical and aligned with the applied load, creating a highly efficient tension element.

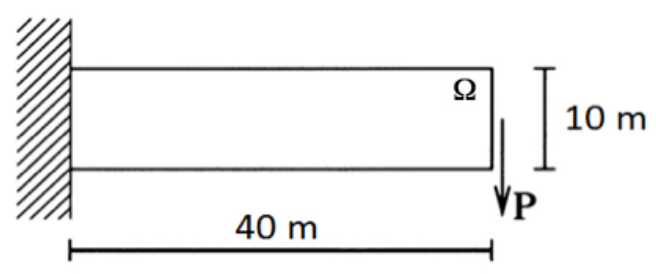

Figure 6. Cantilever beam design domain.
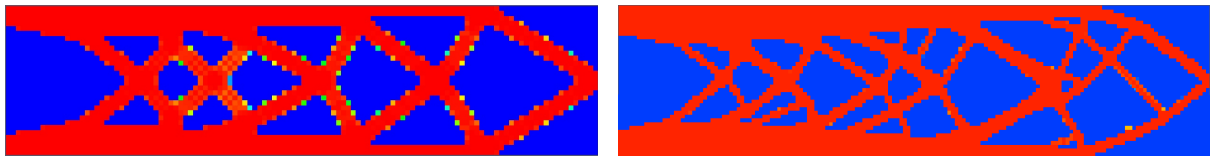

(a) $\operatorname{Load}=\mathrm{P}$
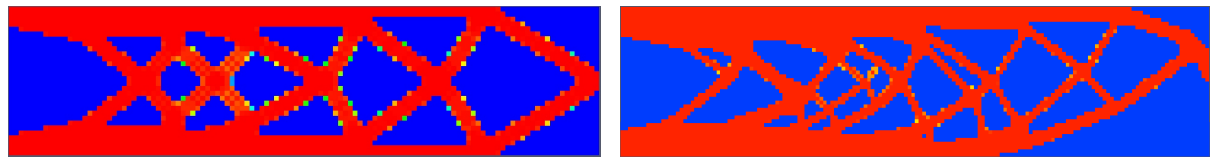

(b) $\mathrm{Load}=2 \mathrm{P}$
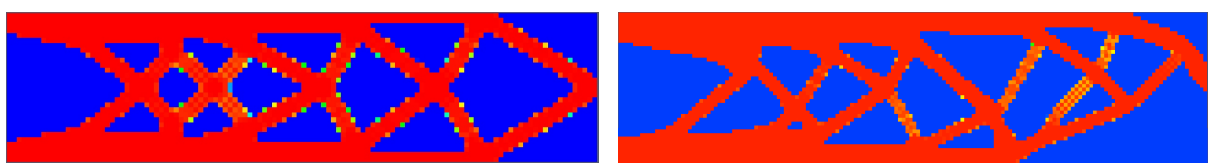

(c) $\operatorname{Load}=2.5 \mathrm{P}$

Figure 7. Cantilever beam topology optimized solutions for various load magnitudes under assumptions of (left) linear mechanics and (right) geometric nonlinearity.

The consideration of material nonlinearities is more complex, as it requires assumptions and sensitivity analysis of post-yield behavior. We use here an elastoplastic model and, following the work of Maute et al. (1998), relate design variable $\rho^{\mathrm{e}}$ to material properties and constitutive tensors following the SIMP logic (equation (2)) as

$$
D=\rho_{i}^{\beta} D_{0} \quad H=\rho_{i}^{\beta} H_{0} \quad \sigma_{y}=\rho_{i}^{\beta} \sigma_{y 0}
$$

where $\mathrm{D}$ is the elastic constitutive matrix, $\mathrm{H}$ is the plastic hardening, $\sigma_{y}$ is the yield stress. For the material nonlinearity, Von-mises Yield surface is used. 


$$
\begin{gathered}
F=\sqrt{3 J_{2}}-k \\
k=\sigma_{y}+H
\end{gathered}
$$

where $J_{2}=\frac{1}{2} S_{i j} S_{j i}, H$ is the hardening and $\sigma_{y}$ is the yield stress.

To demonstrate the effect of including material nonlinearities on final designs, consider the fixed-fixed beam design domain shown in Figure 8. The goal here is to minimize cumulative strain energy under a prescribed midspan deflection of $0.2 \mathrm{~m}$. According to symmetry properties of the example, only one half of the structure is solved.

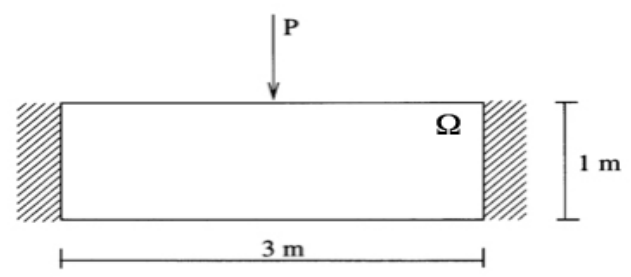

Figure 8. Fixed-fixed beam design domain.

The optimized topology under linear elastic and elastoplastic material models are shown in Figure 9, superimposed on the load-deflection response for each strcutre. The topology resulting under the elastic-plastic material model diversifies the load path resulting in a lower stiffness in the elastic regime but higher stiffness in the nonlinear regime due to the structural redundancies, as the plot demonstrates.

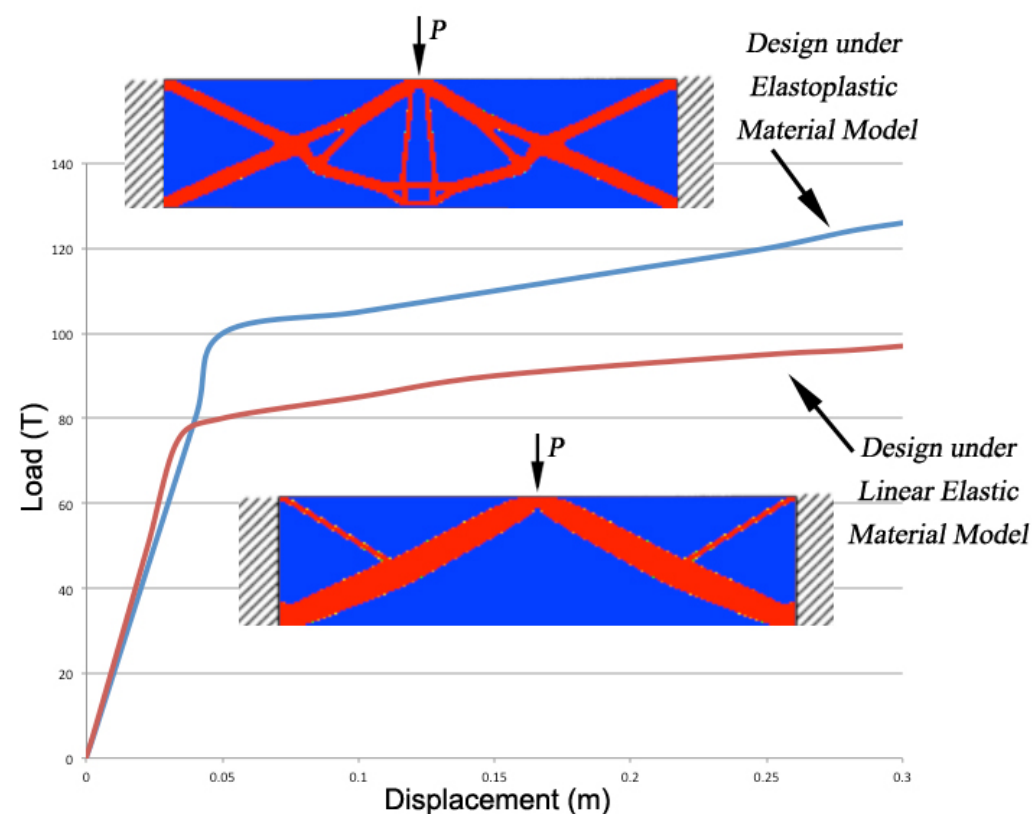

Figure 9. Load-deflection curves for topology optimized beams under conditions of elastic material model and elastic-plastic material model. 


\section{DESIGN UNDER UNCERTAINTY}

Uncertainty in applied loads is often a governing issue in the design of civil engineering structures. Formal incorporation of such randomness into the design optimization framework has recently become a strong focus of the topology optimization community (e.g., Kharmanda et al. 2004, Nguyen et al. in press). Receiving less attention has been consideration of uncertainties in structural stiffness. Such uncertainties arise due to construction and fabrication errors, such as geometric imperfections, construction misfit, and member misalignment, or environmental degradation and damage. The effect of such errors or damage is amplified in structures that are designed using deterministic structural optimization, as such techniques usually produce designs consisting of slender members and structural systems.

The challenge of incorporating random structural stiffness is that the structural response (displacements) are a function of the inverse of a random matrix. This makes the use of simulation-based strategies, such as Monte Carlo simulation, computationally inefficient as each realization results in a different global stiffness matrix (and thus linear system) that must be solved. A key focus of the authors has been in developing intrusive methods that couple the uncertainty quantification with the design sensitivity analysis. These have included the use of perturbation (Guest and Igusa, 2008; Asadpoure et al., 2011) and Polynomial Chaos expansion (Tootkaboni et al., 2012) to optimize structures that are robust in the presence of uncertainty. The robust formulation replaces a deflection metric, for example, with a probabilistic formulation composed of the expected (mean) value of the deflection plus the standard deviation of deflection as follows:

$$
\min \mathrm{E}[d]+k \operatorname{std}[d]
$$

where $\mathrm{E}[\cdot]$ and $\operatorname{std}[\cdot]$ are the expected value and standard deviation operators, respectively, and $k$ is a designer-selected positive scalar that indicates the importance of controlling response variability (with larger $k$ meaning stricter control on response variability).

Of particular interest to the design of structures is the recent coupling of stability analysis with the perturbation-based optimization under uncertain stiffness methodology. Jalalpour et al. (2011) proposed an algorithmic modification that mathematically accounts for the fact that compression amplifies the effect of geometric imperfections while tension dampens it. This led to optimized designs that were dependent on the uncertainty source distribution, the applied load direction, and the applied load magnitude. The work considered mean stiffness only $(k=0)$, which we extend here to consider robust design objectives with $k>0$.

To illustrate the effectiveness of the formulation, we optimize the cantilever truss structure shown in Figure 10 while considering 5\% randomness in all nodal locations to represent the geometric imperfections. The solutions optimized for mean stiffness only $(k=0)$ and mean plus three standard deviations of stiffness $(k=3)$ considering stability are shown in Figure 10. It is immediately evident that the tensile 
zones are relatively light and sparse, while the compression zones feature diversified and braced load paths to mitigate the imperfection-induced onset of buckling. Therefore, including the provisions for resisting buckling leads to designs that have bracings where it is needed the most. We note this diversification and bracing systems is amplified when the designer increases the importance of structure robustness by increasing the variable $k$.
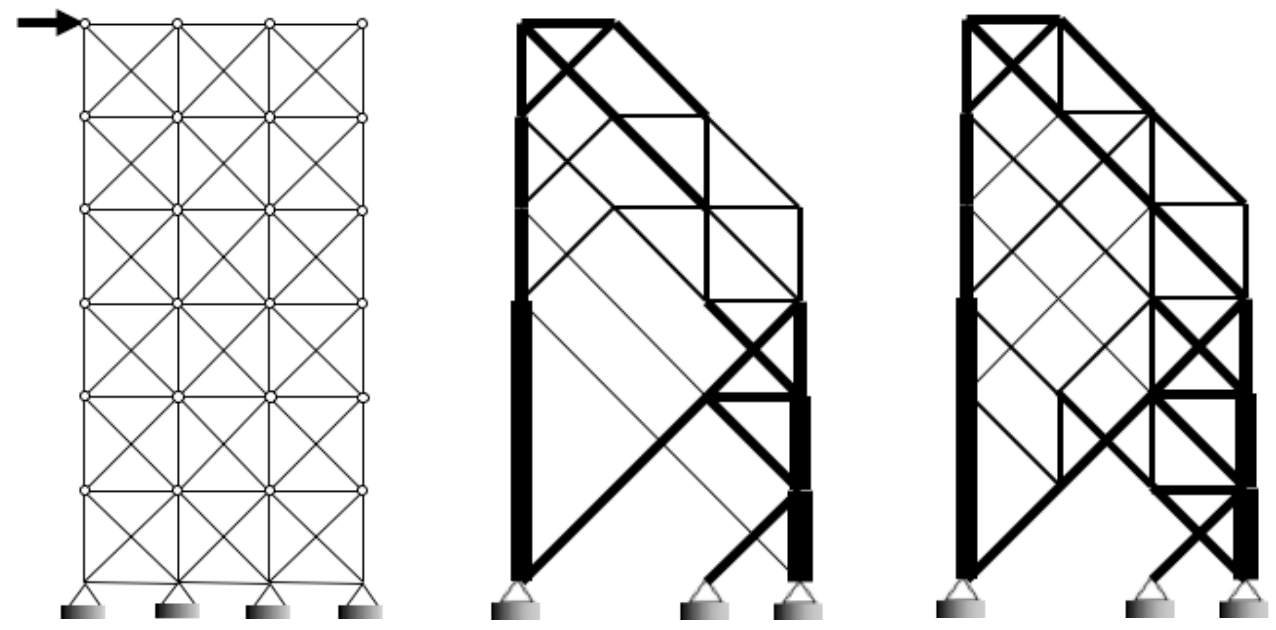

Figure 10. Cantilever design problem (left) and robust topologies optimized for mean stiffness (center) and mean plus three standard deviations of stiffness $(k=3)$ (right) in the presence of geometric imperfections. Considering structural stability leads to diversification and bracing of the compressive load paths.

\section{CONCLUDING REMARKS}

While topology optimization has a demonstrated history of generating new, high performance design ideas, it has generally seen limited, albeit increasing use, by the structural engineering community. The primary reason for this slow adaptation is the reliance of many works on the assumptions (1) that material cost (weight) drives design, and that structural behavior is governed by (2) linear elastic and (3) deterministic mechanics.

This paper summarized recent work by the authors aimed at alleviating these restrictive assumptions, improving the applicability of topology optimization to structural engineering design problems. Specifically, computationally efficient algorithmic tools for (1) influencing the constructability of systems and manufacturability of components, (2) optimizing design under geometric nonlinearities and nonlinear material models, and (3) optimizing robustness of designs under the possibility of construction or fabrication errors, or damage, including the potential stability-induced amplification of such uncertainties. 


\section{ACKNOWLEDGEMENTS}

This work was supported by the National Science Foundation under Grant No. CMMI-0928613 with Dr. Christina Bloebaum serving as program officer. The authors also thank Cris Moen (Virginia Tech) for his input on the strut and tie work and Tak Igusa (JHU) for input on the uncertainty work.

\section{REFERENCES}

Asadpoure, A., Tootkaboni, M., Guest, J.K. (2011). "Robust topology optimization of structures with uncertainties in stiffness - Application to truss structures." Computers \& Structures 89(11-12): 1131-1141.

Bendsøe, M.P. (1989). "Optimal shape design as a material distribution problem." Structural Optimization, 1, 193-202

Bendsøe, M.P., Sigmund, O. (2003). Topology Optimization: Theory, Methods, and Applications, Springer, 2003.

Bruggi, M. (2009). "Generating strut-and-tie patterns for reinforced concrete structures using topology optimization." Computers and Structures, 87(2324), 1483-1495.

Buhl, T., Pederson, C.B.W., Sigmond, O. (2000). "Stiffness design of geometrically nonlinear structures using topology optimization." Structural and Multidisciplinary Optimization, 19(2), 93-104

Darwin, D., Pecknold, D. A. (1977). "Nonlinear biaxial stress-strain law for concrete." ASCE J Eng Mech Div, 103(2), 229-241.

Guest, J.K., Prevost, J.H., Belytschko, T. (2004). "Achieving minimum length scale in topology optimization using nodal design variables and projection functions." International Journal for Numerical Methods in Engineering, 61(2), 238-254.

Guest, J.K., Igusa, T. (2008). "Structural optimization under uncertain loads and nodal locations." Computer Methods in Applied Mechanics and Engineering, 198(1), 116-124.

Guest, J.K. (2009a). "Imposing maximum length scale in topology optimization." Structural and Multidisciplinary Optimization, 37(5): 463-473.

Guest, J.K., (2009b). "Topology optimization with multiple phase projection." Computer Methods in Applied Mechanics and Engineering, 199(1-4), 123135.

Guest, J.K., Moen, C.D. (2010). "Reinforced concrete design with topology optimization." Proceedings of the 19th Analysis and Computation Specialty Conference, 445-454.

Jalalpour, M., Igusa, T., Guest, J.K, (2011). "Optimal design of trusses with geometric imperfections: Accounting for global instability." International Journal of Solids and Structures, 48(21), 3011-3019.

Kharmanda, G., Olhoff, N., Mohamed, A., Lemaire, M. (2004). "Reliability-based topology optimization." Structural and Multidisciplinary Optimization, 26, 295-307. 
Liang, Q.Q., Xie, Y.M., Steven, G.P. (2000). "Topology optimization of strut-and-tie models in reinforced concrete structures using an evolutionary procedure." ACI Structural Journal, 97(2), 322-330.

Maute, K., Schwarz, S., Ramm, E., (1998). "Adaptive topology optimization of elastoplastic structures" Structural and Multidisciplinary Optimization, 15(2), 81-91.

Nguyen, T.H., Song, J., Paulino, G.H. (in press). "Single-loop System ReliabilityBased Topology Optimization Considering Statistical Dependence between Limit-States." Structural and Multidisciplinary Optimization.

Rahmatalla S, Swan C.C. (2003). "Form finding of sparse structures with continuum topology optimization." Journal of Structural Engineering 129:1707-1716.

Schlaich, J., Schaefer, K., Jennewein, M., (1987). "Toward a consistent design of structural concrete." PCI Journal, 32(3), 74-150.

Stromberg, L.L., Beghini, A., Baker, W.F., Paulino, G.H. (2011) "Application of layout and topology optimization using pattern gradation for the conceptual design of buildings. Structural and Multidisciplinary Optimization, 43(2), 165-180.

Svanberg, K., (1987). "The method of moving asymptotes - a new method for structural optimization." International Journal for Numerical Methods in Engineering, 24, 359-373.

Tootkaboni, M., Asadpoure, A., Guest, J.K. (2012). "Topology Optimization of Continuum Structures under Uncertainty - A Polynomial Chaos Approach." Computer Methods in Applied Mechanics and Engineering, 201-204, 263275. 